\title{
S-Adenosyl methionine (SAMe) versus celecoxib for the treatment of osteoarthritis symptoms: A double-blind cross-over trial. [ISRCTN36233495]
}

\author{
Wadie I Najm*1, Sibylle Reinsch², Fred Hoehler ${ }^{2}$, Jerome S Tobis ${ }^{2}$ and \\ Phillip W Harvey ${ }^{2}$
}

Address: ${ }^{1}$ Department of Family Medicine \& Geriatrics, University of California, Irvine, Medical Center, 101 City Drive, Orange, CA 92868, USA and ${ }^{2}$ Department of Physical Medicine \& Rehabilitation, University of California, Irvine, Medical Center, 101 City Drive, Orange, CA 92868, USA

Email: Wadie I Najm* - winajm@uci.edu; Sibylle Reinsch - sreinsch@uci.edu; Fred Hoehler - fhoehler@net-star.net; Jerome S Tobis - jstobis@uci.edu; Phillip W Harvey - pharvey@nnfa.org

* Corresponding author

Published: 26 February 2004

BMC Musculoskeletal Disorders 2004, 5:6

This article is available from: http://www.biomedcentral.com/I47/-2474/5/6

(C) 2004 Najm et al; licensee BioMed Central Ltd. This is an Open Access article: verbatim copying and redistribution of this article are permitted in all media for any purpose, provided this notice is preserved along with the article's original URL.
Received: 22 July 2003
Accepted: 26 February 2004

\begin{abstract}
Background: S-Adenosylmethionine (SAMe) is a dietary supplement used in the management of osteoarthritis (OA) symptoms. Studies evaluating SAMe in the management of OA have been limited to Non Steroidal Anti-inflammatory Drugs (NSAIDs) for comparison. The present study compares the effectiveness of SAMe to a cyclooxygenase-2 (COX-2) inhibitor (celecoxib) for pain control, functional improvement and to decrease side effects in people with osteoarthritis of the knee.

Methods: A randomized double-blind cross-over study, comparing SAMe (1200 mg) with celecoxib (Celebrex $200 \mathrm{mg}$ ) for 16 weeks to reduce pain associated with OA of the knee. Sixtyone adults diagnosed with OA of the knee were enrolled and 56 completed the study. Subjects were tested for pain, functional health, mood status, isometric joint function tests, and side effects.

Results: On the first month of Phase I, celecoxib showed significantly more reduction in pain than SAMe $(p=0.024)$. By the second month of Phase I, there was no significant difference between both groups $(p<0.01)$. The duration of treatment and the interaction of duration with type of treatment were statistically significant ( $p s \leq 0.029)$. On most functional health measures both groups showed a notable improvement from baseline, however no significant difference between SAMe and celecoxib was observed. Isometric joint function tests appeared to be steadily improving over the entire study period regardless of treatment.
\end{abstract}

Conclusion: SAMe has a slower onset of action but is as effective as celecoxib in the management of symptoms of knee osteoarthritis. Longer studies are needed to evaluate the long-term effectiveness of SAMe and the optimal dose to be used.

\section{Background}

Arthritis is one of the most common chronic medical conditions encountered with older age, and osteoarthritis
(OA) is the most common type of arthritis, and a major cause of disability. OA of the knee is more common than any other joint. Symptoms include pain, stiffness, and 
decreased range of motion. Subjects often become limited in their activities and quality of life, due to pain.

To manage the symptoms of OA, subjects and healthcare providers often resort to multiple approaches, which include lifestyle modifications, medications, exercise or surgery. Non-steroidal anti-inflammatory drugs (NSAIDs) are the mainstay medications used to manage osteoarthritis pain. NSAIDs prevent inflammation and control pain by blocking cyclooxygenase (COX-1 and COX-2 enzymes) but their side effect profile is not always acceptable, particularly among the elderly. Each year more than 100,000 subjects are hospitalized for gastrointestinal complications of NSAIDs, and of those approximately 15\% die from these complications [1].

Newer NSAIDs, known as COX-2-inhibitors such as valdecoxib (Bextra), celecoxib (Celebrex), and rofecoxib (Vioxx), were introduced recently as providing similar anti-inflammatory activity and pain relief but fewer gastrointestinal side effects, including a reduced risk of GI erosion and bleeding. However, recent evidence does not always support these claims, and a few studies have suggested a possible increased risk of heart disease [2]. In addition, subjects often lose the benefit from these medications once they are discontinued; however, $\mathrm{OA}$ is a progressive disease and as such requires continuous management.

Over the last decade many dietary supplements were introduced to the public for the management of a variety of conditions including arthritis. Advertisement and information about supplements targeting people with arthritis can be found everywhere. Acupuncture, massage, magnet therapy, and dietary supplements have been promoted for the management of arthritis.

Given the limitations of the established osteoarthritis medications/treatments, and the recent explosion of information and interest in complementary and alternative medicine (CAM), the public has turned their attention to CAM and is exploring safer alternatives to manage their symptoms. The recent American College of Rheumatology (ACR) guidelines for treating OA included dietary supplements, such as glucosamine sulfate, chondroitin sulfate, and antioxidants, as well as acupuncture and magnets as therapies under investigation [3]. While their final judgment of their effectiveness is yet to be scientifically proven, these therapies do seem to have the advantage of showing, possible benefit with fewer side effects. S-Adenosylmethionine (SAMe) is one of the dietary supplements that gained popularity, and was recently reported to be effective in the management of depression, liver disease and arthritis.
SAMe was first discovered in Italy in 1952 [4]. Soon after, it became popular in Europe and more recently in the U.S. SAMe is an important physiologic compound that is distributed throughout the body tissues and fluids. It is produced endogenously from methionine and adenosine triphosphate (ATP). It is an important methyl group donor playing an essential role in many biochemical reactions involving enzymatic transmethylation. Those play an important role in the biosynthesis of phospholipids that are important for the integrity of cell membranes. Despite our basic understanding of its role, the exact mechanism of action in different disease conditions is not well established. Oral SAMe achieves peak plasma concentrations $(0.5-1 \mathrm{mg} / \mathrm{L}) 3$ to 5 hours after ingestion of an enteric-coated tablet $(400-1000 \mathrm{mg})$. The half-life is about 100 minutes, and it is excreted in urine and feces [5].

Biochemically, SAMe is involved in three main metabolic pathways: 1) methylation, as the principal source of methyl groups in the body; 2) transsulfuration, SAMe forms S-Adenosylhomocysteine (SAH) and then converted to homocysteine (Hcy) which can be converted to cystathionine then to cysteine and the sulfate $\left(\mathrm{SO}_{4}\right)$ donated to other metabolic intermediates; and 3) aminopropylation, as SAMe plays an important role in the synthesis of polyamines which can eventually form and recycle methionine [6].

The exact mechanism of SAMe in reducing pain of OA is not known, but evidence suggests that it may play a role in reducing inflammation, increasing proteoglycan synthesis or having an analgesic effect. Whether SAMe is a COX-2 inhibitor is also not known. In vitro studies using human articular chondrocytes have shown SAMe-induced increases in proteoglycan synthesis [7] and proliferation rates in rabbits [8]. SAMe may reduce inflammatory mediators thus reducing pain. This was noted in other studies with the reduction of Tumor Necrosis Factor (TNF)-alpha and fibronectin RNA expression using cultured rabbit synovial cells [9].

Initial studies with SAMe used the parenteral route exclusively due to the instability of the oral form. As additional work allowed the development of a stable oral form of SAMe, further studies tested the effectiveness of the oral form in the management of several medical conditions including osteoarthritis. The length of treatment differed in each study ranging from 7 to 84 days for testing oral preparations, 5 days for intravenous and 7 days for intramuscular preparations. Study designs usually implemented a parallel group design and two studies used a cross-over design [10][11]. Only one cross-over study used an oral preparation with a 5-day washout period [10]. The majority of studies compared SAMe to NSAIDs. 
Most studies used either $600 \mathrm{mg}$ or $1200 \mathrm{mg}$ of SAMe per day. No specific explanations were provided to justify the dose used. The overall conclusions of these studies pointed toward an effect size in favor of SAMe compared to placebo and equivalent to NSAIDs in reducing pain and improving functioning.

Three reviews analyzed available studies to date $[12,13][14]$. One is a meta-analysis [13], the other two provide a general overview of the articles. All three reviews reach similar conclusions that the results of available studies are heterogeneous and do not allow for a firm conclusion about the effectiveness of SAMe in the management of OA. However, despite this limitation the consensus is that SAMe appears to be of equivalent effectiveness to NSAIDs in reducing pain and improving functional limitations, with fewer side effects. Di Padova (1987) [15]concluded that SAMe had a slower onset of action than NSAIDs, with equivalent results at 4 weeks.

Studies were frequently limited though, in quality of design and implementation. Most studies were done during the 1980's, with one study [16] in 1994. Since at that time the majority of the studies compared SAMe to placebo or NSAIDs, there are presently no studies available that evaluate the effectiveness or safety of SAMe versus the new class of drugs used for OA, COX-2 inhibitors. The objective of the present study is to assess the therapeutic effectiveness of oral SAMe (S-adenosylmethionine), in comparison to celecoxib (Celebrex ${ }^{\circledast}$ ), to relieve pain and improve function in adults with OA of the knee.

\section{Methods}

The present study is a prospective randomized double blind cross-over design, of subjects with knee osteoarthritis. We compared the effectiveness of $1200 \mathrm{mg}$ SAMe (600 mg twice daily) with $200 \mathrm{mg}$ celecoxib (Celebrex $100 \mathrm{mg}$ twice daily), to reduce pain and improve functional symptoms associated with OA of the knee. Each phase of the study was for a total of 8 weeks with one-week washout between the two phases.

\section{Setting}

The study was conducted at the General Clinical Research Center (GCRC) in the University of California, Irvine, Medical Center (UCIMC) from September 2000 to September 2002.

\section{Selection criteria}

Inclusion criteria consisted of a) adults age 40 or older, diagnosed with $\mathrm{OA}$ of the knee, based on the American College of Rheumatology criteria for the classification of $\mathrm{OA}$ of the knee, i.e. knee pain morning stiffness up to 30 minutes, and crepitus on motion [17], and b) agreement to participate in the study protocol as described in the informed consent form. Two-view radiographs of the knee that was most affected by OA were obtained. Exclusion criteria consisted of any history of adverse reaction to the study drugs (SAMe, celecoxib or sulfa drugs), current pregnancy status, active infection, blood coagulopathy, use of narcotic analgesics, acute or serious illness, uncontrolled hypertension, moderate to severe $\mathrm{CHF}$, neurological defects involving the lower extremity, bipolar disorder, history of adverse reaction to anti-depressants, or current treatment for depression.

\section{Participants}

Adults with diagnosis of OA of the knee were recruited for this study. Sample size was calculated based on the assumptions of a) a minimum clinically significant difference equal to $10 \%$ of the maximum VAS pain score, b) a population standard deviation of the difference between SAMe and celecoxib equal to $27.5 \%$ of the maximum VAS pain score, c) a two-sided alpha level of 0.05 and d) a beta level of 0.2 ( $80 \%$ power). Using the method given by Lachin [18], 60 patients would be required.

During the open enrollment, four electronic recruitment announcements to all employees at the University of California, Irvine, and its medical center were sent. This resulted in 242 initial inquiries about the study with 190 telephone-screening interviews. Seventy subjects met the inclusion /exclusion criteria and were invited to be examined by the physician. Sixty-one participants agreed and were finally enrolled in this study.

\section{Study design}

The study was a randomized, double-blind, cross-over trial over a 4-month period. Each phase of the study lasted 8 weeks, with one week of washout in between. The Specialty Pharmacy conducted the randomization process after each participant met the inclusion criteria and was enrolled in the study. Allocation to treatment groups was concealed to participants, investigators, and research staff until all the participants had completed the study.

\section{Study procedure}

Participants were assessed in-person during 5 visits conducted at monthly intervals. Visit \#1 was the baseline assessment, visit \#2 was the phase 1 midpoint assessment, visit \# 3 was the end of Phase 1 and served as baseline assessment of Phase 2, visit \#4 was at the midpoint of phase 2, and visit \#5 was at the end of phase 2, upon completion of the study. At visits \#1, 3, and 5, assessments included a review of concurrent medications, a medical examination by a physician and a nurse; assessment of pain, activity impairment, functional health, mood, and knee strength and flexibility measurements. These encounters lasted about 1.5 hours each. At visits \#2 and 4 , assessments included vital signs, weight, edema 
measurements, a review of adverse experiences and pain. Visits \#2 and 4 lasted about 20 minutes each.

During each visit, participants were provided with a calendar page to serve as a health diary for the upcoming month. Participants were asked to note any changes in their health and any additional medication they used for their knee pain. Participants were advised, when possible, to use only acetaminophen (Tylenol) for breakthrough knee pain. When stronger pain medicine was needed, they were asked to note this on their health calendar.

Each participant received a $\$ 100$ honorarium at the end of the study to cover travel expenses for five visits.

\section{Intervention}

Participants were randomly assigned to one of two sequences: Sequence A received SAMe $600 \mathrm{mg}$ bid for 8 weeks in Phase 1, followed by celecoxib (Celebrex) 100 $\mathrm{mg}$ bid for 8 weeks in Phase 2. Sequence B received celecoxib for 8 weeks in Phase 1, followed by SAMe for 8 weeks in Phase 2. One week of washout was allowed prior to dispensing each of the study medicines.

SAMe is biologically synthesized from L-methionine and adenosine triphosphate (ATP) mediated by the enzyme adenosylmethionine synthetase (formally known as methionine adenosyltransferase or MAT) [4]. The SAMe (S-adenosyl-L-methionine disulfate monotosylate salt) used in our study contained $45 \% \mathrm{~S} / \mathrm{S}$ and $55 \% \mathrm{R} / \mathrm{S}$ isomer or a ratio of $45 \% / 55 \%$. The $\mathrm{S} / \mathrm{S}$ or $\mathrm{R} / \mathrm{S}$ indicates the chirality of the amino acid molecule where the first letter refers to the confirmation at the sulfur atom and the second letter refers to the confirmation at the alpha-carbon. A stereospecific colorimetric assay has been shown to identify these isomers [19]. The S-Adenosyl-L-Methionine (SAMe) used in this study was enteric coated $200 \mathrm{mg}$ tablets from 400 mg S-Adenosyl-L-Methionine Disulfate Monotosylate salt, the "elemental" amount of SAMe in the salt mixture is $540 \mathrm{mg}$. The SAMe was verified for quality assurance and potency using a high pressure liquid chromatography (HPLC) analysis with a reference standard of S-adenosylmethionine p-tolunenesulfonate salt (Sigma Lot 77H7053). Results of analysis yielded 99\% of relative label claim with $<0.005 \%$ relative $\%$ of degradation product.

Celecoxib was of pharmaceutical grade quality, available to pharmacies all over the United States. The Research Pharmacy produced "Dummy" placebo capsules and tablets, as celecoxib is marketed in capsules and SAMe in tablets. The SAMe and celecoxib (Celebrex) medications were stored under refrigeration in the research pharmacy, protected from heat and light. Medications were labeled to meet state dispensing requirements and were dispensed in a double-blind, double-dummy randomized design.

\section{Main Outcome Measures}

Pain

A visual analog scale [20] was administered at visits \# $1,2,3,4,5$, with four-week intervals between visits. One scale ascertained pain "today" and a second scale measured pain "in the past month".

\section{Activity impairment}

A 24-item questionnaire, adapted from the Rowland-Morris activity scale [21], was administered at visits \# 1,3, and 5 .

\section{Functional health}

Perceived functional activities were measured with the COOP questionnaire [22], addressing level of physical fitness, emotional distress, daily work, social activity, pain, change in health, overall health, social support, and quality of life. All nine items used pictorial representations and brief descriptions of the five levels of intensity. The COOP questionnaire has been shown to be reliable and valid [23]. The questionnaire was administered at visits \# 1, 3, and 5 .

Functional health was measured with a widely used health survey, the MOS SF-36 [24]. The SF-36 scales include physical functioning, role limitation due to physical health, role limitation due to emotional problems, vitality, mental health, social function, bodily pain, and general health. The SF-36 has been shown to be reliable and valid [23]. The questionnaire was administered at visits \# 1,3, and 5. Both instruments have been shown to have good concurrent validity.

\section{Mood status}

Depression was measured with the short form of the Geriatric Depression Scale [25], comprising 15 yes/no questions. The questionnaire was administered at visits \# 1, 3, and 5.

\section{Clinical assessment of OA of the knee}

A physician, blinded to the participant's randomzation followed the Western Ontario Mac Master (WOMAC) protocol [26] at visits \# 1,3, and 5. Three residents from the Department of Physical Medicine \& Rehabilitation, trained to conduct the protocol, performed clinical assessments to measure tenderness, swelling, and fluid in the knee on a 4-point scale. They measured the knee circumference (in $\mathrm{cm}$ ) at $2 \mathrm{~cm}$ above the superior and $2 \mathrm{~cm}$ below the inferior border of the patella. They asked the participants to indicate the frequency of mild, moderate and severe pain and to rate pain during walking measured on a 10-point scale. They asked participants to indicate 
which activities of daily living they felt impaired, from a choice of 8 activities.

\section{Knee strength and mobility}

An isokinetic multi-joint system, the Biodex System III (Biodex Medical Systems, Shirley, NY), was used to measure isometric strength at 60 degrees (peak flexion and peak extension); isokinetic strength at 60 degrees (peak flexion, peak extension, average flexion, average extension); and isokinetic strength at 180 degrees (peak flexion, peak extension, average flexion, average extension). Walking speed was timed electronically over a 5-meter distance.

\section{Side effects}

Subjects were made aware of possible side effects during the first visit. Adverse reactions were monitored with health diaries, nursing assessments and clinical interviews in-person at visits \# 2,3,4, and 5. Subjects were also asked to phone or e-mail about adverse symptoms.

\section{Statistical methods}

Comparisons between SAMe and celecoxib were based on Analysis of Variance (ANOVA) with factors for treatment (SAMe versus celecoxib), Time (Phase 1 versus Phase 2), Sequence (SAMe-celecoxib versus celecoxib-SAMe) and subjects within sequence. Treatment and time were within-subject effects and Sequence was a between-subjects effect. The primary analysis included only subjects with data from both the SAMe and celecoxib phase. Additional analyses were performed on all available data using the Generalized Estimating Equation (GEE) method to handle missing data.

For pain scores (measured at the middle as well as the end of each treatment period) effects of time on each treatment were analyzed using a within subject factorial ANOVA with effects of treatment (SAMe versus Celecoxib), time on the treatment (Month 1 versus Month 2 ) and the interaction of treatment with time on each treatment.
Comparisons between the two sequence groups at different time periods were performed using Student's t test. Comparisons of post-treatment results with baseline were performed using the paired t test. Comparisons of the incidence of adverse events used the exact binomial test for within-subject comparisons and the Fisher exact test for between-subject comparisons.

Analyses of possible effects of SAMe deterioration on pain scores were analyzed assuming a negative exponential decay function and performing an ANOVA with factors for time on the treatment (Month 1 versus Month 2) and the calculated dose of SAMe.

Statistical comparisons were based on all matched pairs data or on all available data as appropriate. Sample sizes may be lower than the total number of subjects if data were missing. Two-sided p values are reported.

Equivalence tests were performed on pain measures using the Two One-Sided Tests (TOST) procedure [27]. For assessment of equivalence, the minimum clinically significant difference was defined as 10 percent of the maximum VAS pain score. The $90 \%$ confidence intervals were determined using the standard error of the difference scores for all patients with data in both phases of the study.

\section{Results}

\section{Randomization and Compliance}

A total of 61 subjects met the inclusion criteria and were entered in this study (Table 1). After completing the initial assessment, subjects were randomly assigned to sequence A or B. Thirty subjects were randomized to sequence A (SAMe followed by celecoxib) and 31 subjects into sequence B (celecoxib followed by SAMe).

\section{Demographics and Baseline Data}

There were no significant demographic differences between subjects assigned to sequence $\mathrm{A}$ and those assigned to Sequence B (ps > 0.10) (Table 2). Subjects in this study tended to be obese, as indicated by an average body mass index greater than 30 .

Table I: Treatment assignment and compliance.

\begin{tabular}{|c|c|c|}
\hline & Sequence A SAMe - Celecoxib & Sequence B Celecoxib - SAMe \\
\hline Subjects Randomized to each arm & 30 & 31 \\
\hline PHASE I & SAMe & Celecoxib \\
\hline Subjects receiving treatment & 29 & 30 \\
\hline Subjects Completing Phase I & 28 & 29 \\
\hline PHASE 2 & Celecoxib & SAMe \\
\hline Subjects receiving treatment & 28 & 29 \\
\hline Subjects Completing Phase 2 & 27 & 29 \\
\hline
\end{tabular}


Table 2: Demographics and baseline variables.

\begin{tabular}{|c|c|c|c|c|}
\hline \multirow[t]{2}{*}{ Variable } & \multicolumn{2}{|c|}{ Sequence A SAMe - Celecoxib $(\mathbf{N}=28)$} & \multicolumn{2}{|c|}{ Sequence B Celecoxib - SAMe $(N=29)$} \\
\hline & Mean & (SD) & Mean & (SD) \\
\hline Age (years) & 51.6 & $(9.7)$ & 54.1 & $(10.1)$ \\
\hline Height (in) & 66.4 & $(3.9)$ & 66.4 & $(3.5)$ \\
\hline Weight (lbs) & 192.3 & (38.1) & 200.2 & (37.8) \\
\hline \multirow[t]{2}{*}{ Body Mass Index (kg/m2) } & 30.7 & $(5.8)$ & 32.1 & $(6.5)$ \\
\hline & \multicolumn{2}{|c|}{$\mathbf{N}(\%)$} & \multicolumn{2}{|c|}{$\mathbf{N}(\%)$} \\
\hline Females & \multicolumn{2}{|c|}{20 (71.4\%) } & \multicolumn{2}{|c|}{20 (69.0\%) } \\
\hline Males & \multicolumn{2}{|c|}{$8(28.6 \%)$} & \multicolumn{2}{|c|}{$9(31.0 \%)$} \\
\hline White & \multicolumn{2}{|c|}{$21(75.0 \%)$} & \multicolumn{2}{|c|}{$21(72.4 \%)$} \\
\hline Hispanic & \multicolumn{2}{|c|}{5 (17.9\%) } & \multicolumn{2}{|c|}{$5(17.2 \%)$} \\
\hline Asian & \multicolumn{2}{|c|}{$0(0.0 \%)$} & \multicolumn{2}{|c|}{$2(6.9 \%)$} \\
\hline Black & \multicolumn{2}{|c|}{ I (3.6\%) } & \multicolumn{2}{|c|}{ I (3.4\%) } \\
\hline Native American & \multicolumn{2}{|c|}{ I (3.6\%) } & \multicolumn{2}{|c|}{$0(0.0 \%)$} \\
\hline
\end{tabular}

There was no statistically significant difference between the two sequences

Sequence A (SAMe-celecoxib) comprised 30 participants assigned at random, 28 completed Phase 1 of the protocol and 27 completed Phase 2. Twenty-seven participants were analyzed for the primary outcome. Sequence B (celecoxib-SAMe) comprised 31 participants assigned at random, 29 completed Phase 1 of the protocol and 29 completed Phase 2. Twenty-nine participants were analyzed for the primary outcome. Three subjects discontinued participation in Sequence A and two in Sequence B. In each sequence, one subject withdrew during the washout period and one during the celecoxib treatment. During the washout period, the reasons for withdrawal were: insufficient pain to warrant treatment and stroke. During the celecoxib treatment, reasons for withdrawal were: one subject did not reply to follow-up scheduling, and one was diagnosed with rheumatoid arthritis 4 weeks into the treatment. During the SAMe treatment one patient withdrew after 3 days due to headache.

\section{Pain Based on the VAS Scale}

Pain was assessed on two separate visual analog scales. For the first scale, subjects were asked to rate pain today. For the second scale, subjects were asked to rate pain over the past month. Analyses of results from the two measures are similar. In Phase 1, at the end of the first month, the celecoxib group showed significant reductions from baseline (ps $<0.01$ ), while the reductions of pain in the SAMe group were only marginal ( $\mathrm{ps}<0.10$ ). Celecoxib showed significantly more reduction in pain than SAMe during the first month of treatment $(\mathrm{p}=0.024)$. By the second month of Phase 1, both groups were significantly improved from baseline ( $\mathrm{ps}<0.01)$ and there was no significant difference between them.

In Phase 2, during the first month, the group that switched from celecoxib to SAMe was noticeably but not significantly worse than the group that shifted from SAMe to celecoxib. However, by the end of Phase 2, there was no apparent difference between the two groups.

Despite these minor differences, the overall results from the two measures on pain in both groups are similar (See Figure 1).

Because the effects of SAMe and celecoxib seem unaffected by the order of treatment, it is reasonable to combine results from Phase 1 and Phase 2. Cross-over analyses indicated that celecoxib was significantly better at Month 1 (ps $\leq 0.023$ ) but there was no significant difference between the two groups at Month 2 . There were no significant effects of time (Phase 1 versus Phase 2) or sequence (A versus B). The analysis combining Month 1 and Month 2 data showed significant effects of treatment for pain over the past month $(\mathrm{p}=0.043)$ but not for pain today ( $\mathrm{p}$ $=0.247)$. For both pain measures, the effects of duration of treatment and the interaction of duration with type of treatment were statistically significant ( $p s \leq 0.029)$ indicating that the patterns shown in Figures 1-4 are reliable.

The mean difference between the two groups (SAMe minus Celecoxib) on the "pain today" measure was 9.3 at the end of Month 1 and -0.4 at the end of month 2 . The difference score on the "pain over the past month" meas- 


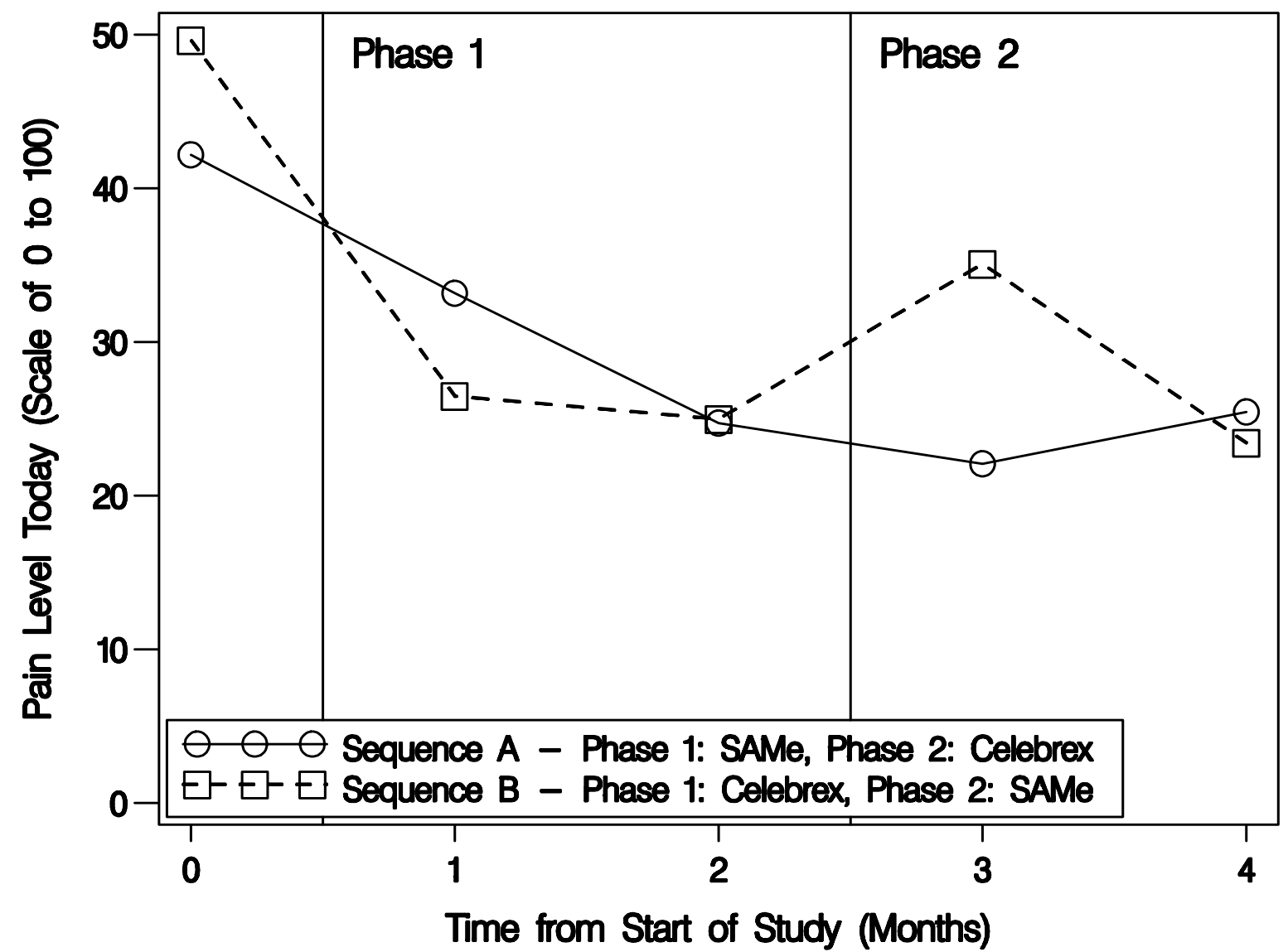

Figure I

Mean VAS pain scores (based on the "pain today" measure) in the two treatment sequence groups. In both sequence groups, SAMe was associated with higher pain scores during the first month of treatment but this effect disappeared after two months of treatment.

ure was 11.6 at the end of Month 1 and 1.6 at the end of Month 2. The 90\% confidence intervals for the "pain today" measure were 2.7 to 15.8 at the end of Month 1 and -7.4 to 6.6 at the end of Month 2 . The $90 \%$ confidence intervals for the "pain over the past month" measure were 5.2 to 18.1 at the end of Month 1 and -6.1 to 9.3 at the end of Month 2. Thus, regardless of the pain measure used, equivalence (based on a minimum clinically significant difference of 10) was demonstrated at the end of Month 2 but not at the end of Month 1 (Figure 2).

\section{COOP Scores}

COOP scores are shown in Table 3. ANOVA showed no significant difference between SAMe and celecoxib on any measure. Effects of time (Phase 1 versus Phase 2) and sequence were also non significant. Both groups showed a significant improvement from baseline in the total COOP score as well as [work], [pain], [change in health] and [overall health]. Neither group showed a significant change from baseline in [physical fitness], [emotional] and [daily activity]. On the [social support] and [quality of life] measures, the SAMe group showed a significant improvement from baseline (ps $\leq$ 0.018) while the celecoxib group did not.

\section{SF-36 Scores}

SF-36 scores are shown in Table 4. ANOVA showed no significant difference between SAMe and celecoxib on any measure. There was a significant effect of time (Phase 1 versus Phase 2 ) on the vitality measure $(p=0.024)$ but, otherwise, there were no significant time or sequence effects. Both groups showed a significant improvement from baseline on the physical function, role physical, bodily pain, and general physical measures ( $\mathrm{ps} \leq 0.045)$. 


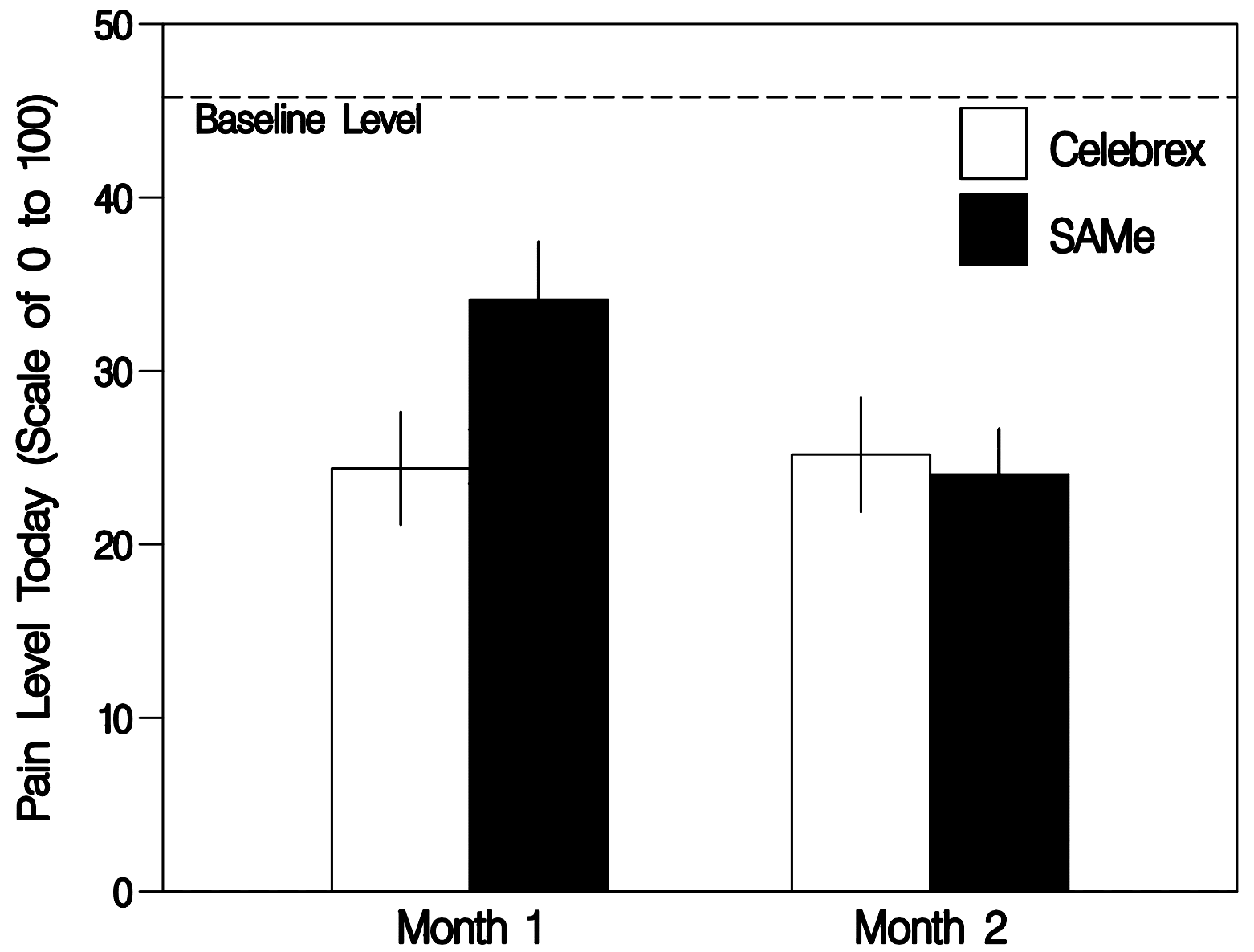

Figure 2

Mean VAS pain scores (based on the "pain today" measure) with standard errors of the mean as a function of treatment with SAMe or Celecoxib.

Neither group showed a significant change from baseline on the role emotional, mental health, social function, general health or general mental measures. The vitality measure showed a significant improvement for the celecoxib group $(\mathrm{p}=0.012)$ but the magnitude of this effect was quite small.

\section{WOMAC, Activity and Mood}

WOMAC scores, Roland-Morris activity scores and Mood scores are shown in Table 5. The only measure that showed any difference between SAMe and celecoxib was circumference of the knee measured in centimeters $(\mathrm{cm})$. The subjects receiving Sequence A showed a decrease from baseline of $0.3 \mathrm{~cm}$ in the first (SAMe) phase and an increase from baseline of $0.2 \mathrm{~cm}$ during the second (celecoxib) phase. The subjects receiving Sequence $\mathrm{B}$ showed an increase from baseline of $0.3 \mathrm{~cm}$ in the first (celecoxib) phase and no change from baseline in the second (SAMe) phase. This difference was statistically significant ( $\mathrm{p}=0.001)$. Curiously, the measures of swelling in the knee and fluid in the knee showed no such trend. The Roland-Morris activity score and the frequency of at least moderate pain showed significant time (Phase 1 versus Phase 2) effects (ps $\leq 0.035$ ) but, otherwise, there were no significant time or sequence effects. Both groups showed significant improvement in tenderness in the knee, swelling in the knee, pain during walking, pain frequency, $\mathrm{ADL}$, the Roland-Morris activity score and the mood score (ps $\leq 0.037$ ). Neither group showed significant changes from baseline in circumference of the knee or swelling of the knee. 
Table 3: Baseline measures of osteoarthritis and related impairment.

\begin{tabular}{|c|c|c|}
\hline \multirow[t]{2}{*}{ Variable } & \multirow{2}{*}{$\begin{array}{c}\text { Sequence A SAMe - Celecoxib }(\mathbf{N}=28) \\
\mathbf{N}(\%)\end{array}$} & \multirow{2}{*}{$\begin{array}{c}\text { Sequence B Celecoxib - SAMe }(\mathbf{N}=29) \\
\mathbf{N}(\%)\end{array}$} \\
\hline & & \\
\hline \multicolumn{3}{|c|}{ Tenderness in Knee* } \\
\hline None & $5(18.5 \%)$ & $6(20.7 \%)$ \\
\hline Slight & $9(33.3 \%)$ & $7(24.1 \%)$ \\
\hline Moderate & $13(48.1 \%)$ & $14(48.3 \%)$ \\
\hline Severe & $0(0.0 \%)$ & $2(6.9 \%)$ \\
\hline \multicolumn{3}{|c|}{ Swelling in Knee* } \\
\hline None & $15(55.6 \%)$ & $6(20.7 \%)$ \\
\hline Slight & $10(37.0 \%)$ & $17(58.6 \%)$ \\
\hline Moderate & $2(7.4 \%)$ & $6(20.7 \%)$ \\
\hline \multicolumn{3}{|c|}{ Fluid in Knee Joint* } \\
\hline None & $22(81.5 \%)$ & $20(69.0 \%)$ \\
\hline Slight & $3(11.1 \%)$ & $7(24.1 \%)$ \\
\hline Moderate & $2(7.4 \%)$ & $2(6.9 \%)$ \\
\hline Redness in Knee* & $0(0.0 \%)$ & I (3.4\%) \\
\hline \multicolumn{3}{|c|}{ Walking Distance** } \\
\hline$<$ । block without pain & $3(10.7 \%)$ & $3(10.3 \%)$ \\
\hline$<$ । block with pain & I (3.6\%) & $4(13.8 \%)$ \\
\hline$<1$ mile without pain & I (3.6\%) & I $(3.4 \%)$ \\
\hline$<1$ mile with pain & $6(21.4 \%)$ & 7 (24.1\%) \\
\hline$>$ I mile without pain & $6(21.4 \%)$ & $4(13.8 \%)$ \\
\hline$>1$ mile with pain & $12(42.9 \%)$ & $13(44.8 \%)$ \\
\hline \multicolumn{3}{|c|}{ Basic ADL - Need Assistance with } \\
\hline Dressing & I (3.6\%) & $0(0.0 \%)$ \\
\hline Transferring & $0(0.0 \%)$ & $0(0.0 \%)$ \\
\hline Bathing & $0(0.0 \%)$ & $0(0.0 \%)$ \\
\hline Toileting & $0(0.0 \%)$ & $0(0.0 \%)$ \\
\hline \multicolumn{3}{|c|}{ Advanced ADL - Difficulty with } \\
\hline Light housework & I (3.6\%) & $4(13.8 \%)$ \\
\hline Heavy housework & $9(32.1 \%)$ & $16(55.2 \%)$ \\
\hline Stooping / crouching & $26(92.9 \%)$ & $27(93.1 \%)$ \\
\hline Getting in/out of car & $17(60.7 \%)$ & $18(62.1 \%)$ \\
\hline
\end{tabular}

* One patient in Sequence A had missing data. ** Multiple responses per patient were allowed. There were no statistically significant differences between the two sequences.

\section{Biodex}

Biodex data are shown in Tables 6 \&8. All of the measures showed significant improvement from baseline (ps $\leq$ 0.001 ) and most of them showed significant time effects (Phase 1 versus Phase 2) in the overall ANOVA. These measures appeared to be steadily improving over the entire study period regardless of treatment. However, there was no significant difference between SAMe and celecoxib.

\section{Adverse Events}

Thirty-six subjects reported adverse events during the SAMe period and 46 subjects reported adverse events during the celecoxib period. The most common adverse 
Table 4: Pain scores (pain was scored on a visual analog scale where $0=$ no pain and $100=$ the highest possible pain level).

\begin{tabular}{|c|c|c|c|c|c|c|c|c|}
\hline \multirow[b]{2}{*}{ Variable } & \multirow[b]{2}{*}{$\mathbf{N}+$} & \multicolumn{2}{|c|}{ Baseline } & \multicolumn{2}{|c|}{ SAMe } & \multicolumn{2}{|c|}{ Celecoxib } & \multirow[b]{2}{*}{ Stdif } \\
\hline & & Mean & (SD) & Mean & (SD) & Mean & (SD) & \\
\hline \multicolumn{9}{|c|}{ Pain Level Today } \\
\hline Month I & 52 & 45.3 & $(23.8)$ & $33.8^{*}$ & $(24.0)$ & $24.6^{*}$ & $(23.9)$ & $-0.33 *$ \\
\hline Month 2 & 52 & 46.1 & $(24.2)$ & $24.0 *$ & $(19.5)$ & $24.4^{*}$ & $(23.6)$ & 0.01 \\
\hline \multicolumn{9}{|c|}{ Pain Level over the Past Month } \\
\hline Month I & 52 & 56.7 & $(22.5)$ & $44.1^{*}$ & $(22.4)$ & $32.5^{*}$ & $(24.0)$ & $-0.42^{* *}$ \\
\hline Month 2 & 52 & 57.0 & $(23.4)$ & $34.7^{*}$ & $(22.7)$ & $33.1^{*}$ & $(22.8)$ & -0.05 \\
\hline
\end{tabular}

Stdif: Standardized difference scores - positive numbers indicate a better response with SAMe. +Sample size is less than 57 due to missing data in either Phase I or Phase 2. *Difference between baseline and treatment is statistically significant $(p<0.05)$. ** Difference between SAMe and Celecoxib is statistically significant $(p<0.05)$.

Table 5: COOP scores.

\begin{tabular}{lcccccccc}
\hline & \multicolumn{4}{c}{ Baseline } & \multicolumn{3}{c}{ SAMe } & \multicolumn{2}{c}{ Celecoxib } \\
\hline Variable & N+ & Mean & (SD) & Mean & (SD) & Mean & (SD) & Stdif. \\
\hline Total Score & 46 & 48.7 & $(8.7)$ & $39.9^{*}$ & $(9.3)$ & $39.8^{*}$ & $(11.3)$ & -0.01 \\
Physical Condition & 46 & 56.2 & $(20.4)$ & 51.7 & $(19.1)$ & 51.3 & $(18.2)$ & -0.02 \\
Emotional Condition & 46 & 37.6 & $(16.1)$ & 33.5 & $(17.9)$ & 34.8 & $(17.6)$ & 0.07 \\
Daily Work & 46 & 47.1 & $(17.0)$ & $34.3^{*}$ & $(14.4)$ & $33.9 *$ & $(15.7)$ & -0.03 \\
Social Activities & 46 & 31.4 & $(15.4)$ & 29.1 & $(13.1)$ & 29.6 & $(15.6)$ & 0.02 \\
Pain & 46 & 70.5 & $(14.1)$ & $53.0^{*}$ & $(15.9)$ & $50.9 *$ & $(19.2)$ & -0.10 \\
Change in Condition & 46 & 65.2 & $(13.3)$ & $49.1^{*}$ & $(16.7)$ & $47.8^{*}$ & $(18.6)$ & -0.05 \\
Overall Condition & 46 & 50.0 & $(16.1)$ & $44.3^{*}$ & $(16.3)$ & $41.3^{*}$ & $(16.0)$ & -0.19 \\
Social Support & 46 & 39.0 & $(24.6)$ & $29.6^{*}$ & $(18.3)$ & 32.6 & $(18.1)$ & 0.17 \\
Quality of Life & 46 & 41.0 & $(13.9)$ & $34.3^{*}$ & $(16.1)$ & 36.1 & $(13.1)$ & 0.11
\end{tabular}

Stdif: Standardized difference scores - positive numbers indicate a better response with SAMe +Sample sizes are less than 57 due to missing data in either Phase I or Phase 2. *Difference between baseline and treatment is statistically significant $(p<0.05)$. No statistically significant differences were observed between SAMe and Celecoxib

events were gastrointestinal disorder (SAMe 4: celecoxib: 6, NS), anxiety (SAMe: 5, celecoxib 4, NS) and dyspepsia (SAMe: 1, celecoxib: 3, NS). No other adverse event was reported by more than two subjects. One patient terminated the study due to headache three days into the SAMe treatment; however this patient had a well known history of such headaches prior to enrolling in the study.

\section{Loss of SAMe potency}

The increased incidence of pain in the SAMe group was concentrated in the first month of Phase 2 where 12 of 29 subjects who had recently switched from celecoxib to SAMe complained of pain, while only 2 of 24 subjects recently switched from SAMe to celecoxib complained of pain $(\mathrm{p}=0.011)$. The difference between groups was not statistically significant at any other time period.

Approximately $75 \%$ of the way through the study, a routine quality check of a random sample of the study medicine indicated that the SAMe had lost approximately 51\% of its potency. At this time, the study was delayed until a new batch of SAMe could be obtained. In order to examine this effect, potency was crudely estimated based on the assumption of an exponential decay curve from $100 \%$ potency at the start of the study to $49 \%$ potency at day 595 . This yields the equation $y=\exp (-0.0011989 x)$ where $\mathrm{y}$ is potency and $\mathrm{x}$ is days from the start of the study or days from the starting time for the new batch of SAMe. An ANOVA on pain-today scores in SAMe treated subjects 
Table 6: SF-36 scores (higher scores indicate less impairment).

\begin{tabular}{lcccccccc}
\hline & \multicolumn{4}{c}{ Baseline } & \multicolumn{3}{c}{ SAMe } & \multicolumn{2}{c}{ Celecoxib } \\
\hline Variable & N+ & Mean & (SD) & Mean & (SD) & Mean & (SD) & Stdif. \\
\hline Physical Function & 53 & 52.5 & $(20.8)$ & $62.4^{*}$ & $(22.8)$ & $63.7^{*}$ & $(25.1)$ & -0.10 \\
Role Physical & 53 & 56.6 & $(37.4)$ & $66.5^{*}$ & $(33.6)$ & $67.9^{*}$ & $(38.1)$ & -0.04 \\
Role Emotional & 53 & 86.8 & $(29.5)$ & 88.7 & $(24.4)$ & 87.4 & $(27.1)$ & 0.06 \\
Vitality & 53 & 58.9 & $(14.4)$ & 60.4 & $(14.0)$ & $62.9 *$ & $(14.5)$ & -0.23 \\
Mental Health & 53 & 71.8 & $(11.4)$ & 73.7 & $(11.3)$ & 74.0 & $(11.8)$ & -0.03 \\
Social Function & 53 & 82.3 & $(20.1)$ & 84.2 & $(19.8)$ & 86.3 & $(18.7)$ & -0.09 \\
Bodily Pain & 53 & 46.8 & $(14.1)$ & $59.3^{*}$ & $(19.8)$ & $63.1^{*}$ & $(19.6)$ & -0.16 \\
General Health & 53 & 74.6 & $(15.2)$ & 75.4 & $(17.2)$ & 76.6 & $(16.8)$ & -0.10 \\
General Physical & 53 & 38.1 & $(8.2)$ & $42.3^{*}$ & $(9.5)$ & $43.4^{*}$ & $(10.6)$ & -0.16 \\
General Mental & 53 & 54.6 & $(7.8)$ & 54.1 & $(7.4)$ & 54.2 & $(6.8)$ & -0.02 \\
\hline
\end{tabular}

Stdif: Standardized difference scores - positive numbers indicate a better response with SAMe. +Sample sizes are less than 57 due to missing data in either Phase I or Phase 2. *Difference between baseline and treatment is statistically significant $(\mathrm{p}<0.05)$. No statistically significant differences were observed between SAMe and Celecoxib.

with a factor for duration of treatment ( 1 month versus 2 months) and a continuous potency factor produced a significant effect of month showing improvement from Month 1 to Month 2 ( $p<0.001)$. The effect of SAMe potency was in the expected direction (lower estimated potency was correlated with higher pain) but the effect was not statistically significant $(\mathrm{p}=0.188)$.

\section{Discussion}

To our knowledge this is the first study comparing the effectiveness of SAMe to a COX-2 inhibitor in the management of osteoarthritis symptoms. Recent studies have demonstrated that COX-2 inhibitors have equivalent effectiveness but a better safety profile than other NSAIDs in the management of osteoarthritis. Their advantage is that by avoiding the COX-1 inhibitors they would have less tendency to produce gastrointestinal side effects. Published studies compared SAMe most commonly to 1200 mg of ibuprofen. Despite its efficacy at $1200 \mathrm{mg}$, ibuprofen is more frequently dispensed at $1600 \mathrm{mg}$ /day, or higher, based on the underlying conditions. To avoid any doubt or controversy, we opted to use the most frequently used dose of SAMe and the optimal dose of celecoxib. Future studies should explore different doses of SAMe, to identify the lowest most effective and safest dose to be used.

Our results indicate that SAMe is equivalent in almost all measures to COX-2 inhibitors (celecoxib) in relieving pain and improving function in subjects with osteoarthritis of the knee. It is clear from our results that SAMe has a slower onset of action, requiring almost one month of treatment prior to achieving similar effect to celecoxib. COX-2 inhibitors and NSAIDs have a definite advantage during the first month of treatment. This is consistent with prior conclusions by di Padova [15]. However, it does not clearly explain findings of several previously published small studies [28][29][30][31]. These studies although limited to 1 month of duration, reported equivalent effectiveness to NSAIDs in relieving symptoms of OA.

During the second month of each phase of the study, the pain relieving effect is equivalent for both drugs. Of interest is that, while the pain relief of celecoxib was constant throughout the study, the effect of SAMe continued to increase with time (Figure 1). This raises the question whether the effect of SAMe would have continued to improve had the study been for a longer period of time? Hence a more extended study is needed to evaluate the long-term effect of SAMe and to assess the presence of a possible ceiling effect of this supplement. Another possible interpretation could be that the effect of SAMe slows the progression of the disease, hence the persistent effect after discontinuation. The same effect was not seen with celecoxib.

Although this study was not set up to evaluate the longterm effect of SAMe, the data hints that the pain relief effect obtained with SAMe persisted even after the medication was discontinued (Figure 1). In this study we noted that subjects who changed from SAMe to celecoxib had an initial decline in their pain level, however the level was back to baseline at 2 months consistent with the level of those that were started on celecoxib. Although the difference was not statistically different in both groups, this initial dip beyond the baseline pain relief obtained by celecoxib alone hint for a possible persistent effect of SAMe for 1 month beyond discontinuation of the medication. This is reminiscent of reports on the pain relieving 
Table 7: WOMAC, activity and mood scores (higher scores indicate more impairment: scaled from 0 to 100 except for knee circumference).

\begin{tabular}{|c|c|c|c|c|c|c|c|c|}
\hline \multirow[b]{2}{*}{ Variable } & \multirow[b]{2}{*}{$\mathbf{N +}$} & \multicolumn{2}{|c|}{ Baseline } & \multicolumn{2}{|c|}{ SAMe } & \multicolumn{2}{|c|}{ Celecoxib } & \multirow[b]{2}{*}{ Stdif. } \\
\hline & & Mean & (SD) & Mean & (SD) & Mean & (SD) & \\
\hline \multicolumn{9}{|c|}{ WOMAC Knee Assessment } \\
\hline Tenderness & 50 & 29.9 & $(21.8)$ & $14.7^{*}$ & $(17.4)$ & 19.3* & $(18.9)$ & 0.21 \\
\hline Swelling & 51 & 15.7 & (15.6) & $9.8^{*}$ & (14.6) & $8.2^{*}$ & $(12.2)$ & -0.11 \\
\hline Fluid & 49 & 6.6 & $(14.1)$ & 6.8 & $(14.8)$ & 4.4 & $(10.1)$ & -0.14 \\
\hline \multicolumn{9}{|c|}{ Knee Circumference (cm) } \\
\hline Total & 50 & 41.6 & $(4.8)$ & 41.5 & $(4.7)$ & 42.1 & $(5.0)$ & $0.52^{* *}$ \\
\hline Above patella & 50 & 45.0 & $(6.0)$ & 44.8 & (5.7) & 45.4 & (5.7) & $0.43 * *$ \\
\hline Below patella & 50 & 38.3 & (3.7) & 38.2 & (3.8) & $38.9 *$ & $(4.5)$ & $0.40 * *$ \\
\hline \multicolumn{9}{|c|}{ WOMAC Pain Frequency } \\
\hline Mild & 49 & 73.3 & $(27.9)$ & $57.1^{*}$ & $(28.4)$ & $61.2^{*}$ & $(27.0)$ & 0.12 \\
\hline Moderate & 46 & 65.6 & $(19.4)$ & $46.2^{*}$ & $(25.8)$ & $38.6 *$ & $(24.6)$ & -0.24 \\
\hline Severe & 46 & 45.7 & $(20.8)$ & $26.6^{*}$ & $(22.6)$ & $22.8^{*}$ & $(24.1)$ & -0.14 \\
\hline \multicolumn{9}{|c|}{ WOMAC Other } \\
\hline Pain - Walking & 25 & 37.8 & $(31.3)$ & $17.6^{*}$ & $(20.5)$ & 19.2* & $(22.7)$ & 0.07 \\
\hline ADL & 50 & 26.0 & $(13.0)$ & $20.3^{*}$ & $(14.2)$ & $17.8^{*}$ & $(15.2)$ & -0.19 \\
\hline Activity Score & 53 & 55.6 & $(24.3)$ & $37.0 *$ & $(27.4)$ & $34.8^{*}$ & $(30.0)$ & -0.10 \\
\hline Mood Score & 52 & 13.2 & $(14.8)$ & $8.6^{*}$ & (II.8) & $9.9 *$ & $(12.2)$ & 0.17 \\
\hline
\end{tabular}

Stdif: Standardized difference scores - positive numbers indicate a better response with SAMe. +Sample sizes are less than 57 due to missing data in either Phase I or Phase 2. *Difference between baseline and treatment is statistically significant $(p<0.05)$. ** Difference between SAMe and Celecoxib is statistically significant $(p<0.05)$.

effect observed with glucosamine sulfate. If this result could be reproduced in future large studies, the question will be raised of whether SAMe could be used as a pulsed therapy, for the management of pain in OA, after an initial period to establish a steady level?

\section{Functional health}

Several health measures were assessed during this study, including medical and joint function assessments, measures of daily function and activity impairment, assessments of mood and quality of life. On most of these measures, both treatment groups showed significant improvements from baseline and isometric joint function tests appeared to be steadily improving over the entire study period as well, regardless of treatment. No statistically significant difference was seen between SAMe and celecoxib. Thus, the evidence of comparable efficacy between SAMe and celecoxib seems rather solid.

Of interest but of unclear clinical significance is the measurement of the knee circumference. The circumference dif- fered significantly between the two treatments, with a decrease from baseline of $0.3 \mathrm{~cm}$ during the SAMe phase and an increase from baseline of $0.2 \mathrm{~cm}$ during the celecoxib phase. While this statistical difference does not seem to have much clinical meaning, future studies on the mechanism of action of SAMe might shed light on this decrease in knee circumference.

Perceived physical fitness, emotional well-being and daily activities did not improve from baseline with either treatment. Fitness and well-being as well as daily activities might represent more stable attributes than physical complaints and thus might not change in a 2-months treatment.

\section{Depression}

Of interest is that no participant in this study was identified with depression, using the abbreviated geriatric depression scale, during this study. Hence the results and discussion of effects of SAMe on depression in this study 
Table 8: Biodex data (except for walking speed, higher scores indicate less impairment).

\begin{tabular}{|c|c|c|c|c|c|c|c|c|}
\hline \multirow[b]{2}{*}{ Variable } & \multirow[b]{2}{*}{$\mathbf{N}+$} & \multicolumn{2}{|c|}{ Baseline } & \multicolumn{2}{|c|}{ SAMe } & \multicolumn{2}{|c|}{ Celecoxib } & \multirow[b]{2}{*}{ Stdif. } \\
\hline & & Mean & (SD) & Mean & (SD) & Mean & (SD) & \\
\hline \multicolumn{9}{|c|}{ Isometric 60 degrees } \\
\hline Peak flexion & 45 & 66.7 & $(30.6)$ & $80.2^{*}$ & $(30.0)$ & $77.5^{*}$ & $(29.2)$ & 0.21 \\
\hline Peak extension & 45 & 92.4 & $(40.8)$ & $116.6^{*}$ & $(52.0)$ & $112.5^{*}$ & $(50.3)$ & 0.19 \\
\hline \multicolumn{9}{|l|}{ Isokinetic 60 degrees } \\
\hline Peak flexion & 45 & 40.5 & $(2 \mid .2)$ & $54.8^{*}$ & $(26.5)$ & $52.3^{*}$ & (20.9) & 0.16 \\
\hline Peak extension & 45 & 84.2 & $(43.5)$ & $107.1^{*}$ & $(43.0)$ & $102.4^{*}$ & $(39.0)$ & 0.24 \\
\hline Average flexion & 45 & 22.2 & $(16.8)$ & $32.1 *$ & $(18.4)$ & $29.9 *$ & $(15.2)$ & 0.19 \\
\hline Average extension & 45 & 48.0 & $(27.5)$ & $64.3^{*}$ & (28.3) & $62.3^{*}$ & $(26.4)$ & 0.14 \\
\hline \multicolumn{9}{|c|}{ Isokinetic 180 degrees } \\
\hline Peak flexion & 45 & 30.7 & $(15.7)$ & $39.0 *$ & $(16.3)$ & $37.9 *$ & $(15.1)$ & 0.13 \\
\hline Peak extension & 45 & 57.0 & $(29.5)$ & $72.3^{*}$ & $(31.0)$ & $69.3^{*}$ & $(28.0)$ & 0.19 \\
\hline Average flexion & 45 & 25.3 & $(27.8)$ & $42.7^{*}$ & (32.4) & $39.3^{*}$ & $(29.3)$ & 0.18 \\
\hline Average extension & 45 & 76.5 & $(53.0)$ & $109.6 *$ & (56.4) & $105.6^{*}$ & $(50.9)$ & 0.14 \\
\hline Walking Speed & 42 & 3.12 & (1.52) & $2.63^{*}$ & $(0.91)$ & $2.56^{*}$ & $(0.69)$ & -0.14 \\
\hline
\end{tabular}

Stdif: Standardized difference scores - positive numbers indicate a better response with SAMe. +Sample sizes are less than 57 due to missing data in either Phase I or Phase 2. *Difference between baseline and treatment is statistically significant $(P<0.05)$. No statistically significant differences were observed between SAMe and Celecoxib.

should be interpreted with caution and could not be generalized to a depressed population.

Despite a slight drop in the mood scale while subjects were on SAMe (see Table 7), no statistical difference in the depression scale was noted while subjects were on SAMe during this study. However, one conclusion remains clear, given the absence of positive antidepressive effects seen in this study, one can conclude that the beneficial results (pain, function) noted in this study could not be explained by the antidepressive effect of SAMe. Hence other mechanisms of action are in effect, and further studies are warranted to explore these mechanism.

\section{Adverse events}

The type of side effects seen in this study is consistent with those reported in other trials. Gastrointestinal, psychiatric, insomnia, allergy, and rash were all reported in previous studies. Again consistent with prior data, gastrointestinal and psychiatric side effects were the most common. This is consistent with findings by Soeken et al. [13] that subjects on SAMe were less likely to experience side effects than those treated with NSAIDs. In her analysis, Soeken reports that this is independent of SAMe dose or length of intervention.
Of note is that the most common reported complaint in this study, although not included under adverse event, is pain. This could be attributed to either failure of the medication to provide adequate pain relief (SAMe during the first weeks of therapy); or for this study during the period where the potency of SAMe tablets was initially noted to have declined.

\section{Potency and quality assurance}

SAMe is produced biologically in the $\mathrm{S} / \mathrm{S}$ form. Both $\mathrm{R} / \mathrm{S}$ and $\mathrm{S} / \mathrm{S}$ isomers are biologically active [32]. Under normal physiological conditions or normal storage conditions, SAMe spontaneously racemizes to form a mixture of $\mathrm{S}$ and $\mathrm{R}$ isomers, thus over time, the $\mathrm{S} / \mathrm{S}$ form converts into the $\mathrm{R} / \mathrm{S}$ form. This is an equilibration phenomenon and occurs in the commercial form. The racemisation at the sulfur side of the SAMe molecule has been established in the literature [33]. All commercial SAMe products are manufactured by combining fermentation and synthetic steps. Commercially available SAMe has been tested to have different ratios of $\mathrm{S} / \mathrm{S} \& \mathrm{R} / \mathrm{S}$ isomers ranging from $70 \% / 30 \%$ to $45 \% / 55 \%$ (unpublished data). Several studies have indicated that the S/S-SAMe configuration is the "active" form involved in methyltransferase catalyzed reactions [34]'[35]'[36] and that the R/S-SAMe configuration is the biologically "inactive" form as it may inhibit methyltransferases reactions [37]. In more of a functional 
outcome, other authors have shown that both S/S and R/ $S$ isomers have significant activity respectively in increasing blood flow and bile production in isolated perfused rat livers [38]. Therefore, there may not appear to be any difference in the biological effects of the different ratios of SAMe.

In this study, we did not perform analysis of the S/S \& S/ $\mathrm{R}$ isomers during quality assurance. Hence we are unable to verify whether formulations with higher or lower S/S or $\mathrm{S} / \mathrm{R}$ isomers are more potent in the management of OA. Our experience highlights the need for future studies, involving dietary supplements, to develop a quality assurance safeguard.

Given the absence of clear and well established explanation for the mechanism of action for SAMe in the management of OA, future studies should explore the possible role of isomers.

\section{Limitations of the study}

Although this study had fewer patients than originally proposed, it retained sufficient power to conclude that a) celecoxib is significantly better than SAMe in the first month of treatment and b) by the second month of treatment, SAMe and celecoxib were clinically equivalent based on the proposed equivalence criterion.

Findings should be interpreted with caution due to the small number of participants and the short duration of the study. Toward the end of the study, SAMe lost about half of its potency and the study was delayed until a new supply of SAMe was obtained. While the lower potency was associated with higher pain, this effect was non-significant and did not affect the overall study outcome. Future long-term studies evaluating dietary supplements should implement quality checks at appropriate time intervals for adequate quality assurance.

\section{Conclusion}

SAMe is helpful for the management of pain in osteoarthritis, and demonstrates similar effectiveness as a currently accepted COX-2 inhibitor celecoxib. Results from this study add and confirm results from prior studies indicating a possible role for SAMe in the management of osteoarthritis. It is clear however, that many questions remain unanswered. Prime among these questions is: What is the optimal (effective and safe) dose SAMe in the management of osteoarthritis? Other question that should be explored are: What is the long-term effectiveness and safety of SAMe? What is the mechanism of action of SAMe? Does SAMe effect the disease progression or could it reverse the disease process? and finally Is the combination of SAMe and a COX-2 inhibitor more effective than either alone in the management of osteoar- thritis? Most of the currently available studies offer a promise, however well done randomized controlled trials are needed to answer any and all of these questions.

\section{Competing interests}

None declared.

\section{Authors' contributions}

JST, SR, and WIN conceived the initial research design and protocol. SR and JST carried out data collection and dayto-day coordination of this study. FH provided all the statistical and methodological support. PWH provided advice and coordinated the procurement of SAMe. All the authors contributed to the manuscript and approved the final manuscript.

\section{Acknowledgement}

The authors wish to thank the Susan Samueli Center of Integrative Medicine at the University of California, Irvine ( $\mathrm{UCl}$ ) for funding this study. We would also like to acknowledge the staff at the UCI General Clinical Research Center for providing logistic and technical support, and NOW ${ }^{\circledR}$ Foods for providing SAMe for this study.

We also wish to acknowledge and thank Larry Plon, PharmD for providing pharmacy support, and Drs. Ling Shi-Bertsch and Venus Ramos (PM\&R residents at UC Irvine) for the medical evaluation and follow-up of participants.

\section{References}

I. Singh G, Triadalfilopolous G: Epidemiology of NSAID induced GI complications. J Rheumatol 1999, 26:18-24.

2. Lane NE: Pain management in osteoarthritis: the role of COX-2 inhibitors. J Rheumatol 1997, 24:20-24.

3. Recommendations for the medical management of osteoarthritis of the hip and knee: 2000 update. American College of Rheumatology Subcommittee on Osteoarthritis Guidelines. Arthritis Rheum 2000, 43:1905-1915.

4. Cantoni GL: The nature of active methyl donor formed enzymatically from L-methionine and adenosinetriphosphate. J Am Chem Soc 1952, 74:2942-2943.

5. Friedel HA, Goa KL, Benfield P: S-adenosyl-L-methionine. A review of its pharmacological properties and therapeutic potential in liver dysfunction and affective disorders in relation to its physiological role in cell metabolism. Drugs 1989, 38:389-416.

6. Bottiglieri T: S-Adenosyl-L-methionine (SAMe): from the bench to the bedside- molecular basis of a pleiotrophic molecule. Am J Clin Nutr 2002, 76: I I5IS-7S.

7. Harmand MF, Vilamitjana J, Maloche E, Duphil R, Ducassou D: Effects of S-adenosylmethionine on human articular chondrocyte differentiation. An in vitro study. Am J Med 1987, 83:48-54.

8. Barcelo HA, Wiemeyer JC, Sagasta CL, Macias M, Barreira JC: Effect of S-adenosylmethionine on experimental osteoarthritis in rabbits. Am J Med 1987, 83:55-59.

9. Gutierrez S, Palacios I, Sanchez-Pernaute O, al et: SAMe restores the changes in the proliferation and in the synthesis of fibronectin and proteoglycans induced by tumour necrosis factor alpha on cultured rabbit synovial cells. $\mathrm{Br} J$ Rheumatol 1997, 36:27-31.

10. Pellegrini P: [S-adenosylmethionine (SAMe) in osteoarthrosis; a double-blind crossover peroral study]. G Clin Med 1980, 61:616-627.

II. Polli E, Cortellaro M, Parrini L, Tessari L, Cherie Ligniere G: [Pharmacological and clinical aspects of S-adenosylmethionine (SAMe) in primary degenerative arthropathy (osteoarthrosis)]. Minerva Med 1975, 66:4443-4459. 
12. di Padova C: S-Adenosylmethinine in the treatment of osteoarthritis. Review of the clinical studies. Am. J Med 1987, 83:60-65.

13. Soeken KL, Lee WL, Bausell RB, Agelli M, Berman BM: Safety and efficacy of S-adenosylmethionine (SAMe) for osteoarthritis. J Fam Pract 2002, $51: 425-430$.

14. Center Southern California Evidence-Based Practice: S-Adenosyl-LMethionine (SAMe) for Depression, Osteoarthritis, and Liver Disease. Evidence Report/Technology Assessment: Number 64.2002 [http://www.ahrq.gov/clinic/evrptfiles.htm\#same.]. Agency for Healthcare Research and Quality

15. di Padova C: S-adenosylmethionine in the treatment of osteoarthritis. Review of the clinical studies. Am J Med 1987, 83:60-65.

16. Bradley JD, Flusser D, Katz BP, Schumacher H. R., Jr., Brandt KD, Chambers MA, Zonay LJ: A randomized, double blind, placebo controlled trial of intravenous loading with S-adenosylmethionine (SAM) followed by oral SAM therapy in patients with knee osteoarthritis. J Rheumatol 1994, 21:905-91 I.

17. Hochberg MC, Kasper J, Williamson J, Skinner A, Fried LP: The contribution of osteoarthritis to disability: preliminary data from the Women's Health and Aging Study. J Rheumatol Suppl $1995,43: 16-18$.

18. Lachin JM: Introduction to sample size determination and power analysis for clinical trials. Control Clin Trials 1981, 2:93-113.

19. Cannon LM, Butler FN, Wan W, Sunny Zhou Z: A stereospecific colorimetric assay for $(\mathbf{S}, \mathbf{S})$-adenosylmethionine quantification based on thiopurine methyltransferase-catalyzed thio methylation. Anal Biochem 2002, 308:358-363.

20. Scott J, Huskisson EC: Graphic representation of pain. Pain 1976, 2:175-184

21. Roland M, Morris R: A study of the natural history of back pain: Part I. Development of a reliable and sensitive measure of disability in low-back pain. Spine 1994, 19:257|-2577.

22. Nelson EC, Landgraf JM, Hays RD, Wasson JH, Kirk JW: The functional status of patients. How can it be measured in physicians' offices? Med Care 1990, 28: I I I I- I 26.

23. Bronfort G, Bouter LM: Responsiveness of general health status in chronic low back pain: A comparison of the COOP Charts and the SF-36. Pain 1999, 83:20I-209.

24. Ware JE Jr., Sherbourne CD: The MOS 36-Item Short-Form Health Survey (SF-36): Conceptual framework and item selection. Med Care 1992, 30:473-483.

25. Sheikh JI, Yesavage JA: Geriatric Depression Scale (GDS): Recent evidence and development of a shorter version. Clinical Gerontology : A Guide to Assessment and Intervention Edited by: Sheikh JI and Yesavage JA. New York, The Haworth Press; 1986:165-173.

26. Bellamy N, Buchanan W, Goldsmith C, Campbell J, L. Stitt: Validation Study of WOMAC: A Health Status Instrument for Measuring Clinically Important Relevant Outcomes to Antirheumatic Drug Therapy in Patients with Osteoarthritis of the Hip or Knee. J Rheumatology 1988, I 5: I 833-1840.

27. Schuirmann DJ: A comparison of the two one-sided tests procedure and the power approach for assessing the equivalence of average bioavailability. J Pharmacokinet and Biopharm 1987, 15:657-680.

28. Vetter G: Double-blind comparative clinical trial with S-adenosylmethionine and indomethacin in the treatment of osteoarthritis. Am J Med 1987, 83:78-80.

29. Muller-Fassbender H: Double-blind clinical trial of S-adenosylmethionine versus ibuprofen in the treatment of osteoarthritis. Am J Med 1987, 83:8I-83.

30. Caruso I, Pietrogrande V: Italian double-blind multicenter study comparing S-adenosylmethionine, naproxen, and placebo in the treatment of degenerative joint disease. Am J Med 1987, 83:66-7I.

31. Cucinotta D, Mancini M, Ceccato S, Castino E: [Controlled clinical study of SAMe (S-adenosylmethionine) administered orally in degenerative osteoarticular pathology]. G Clin Med 1980, 6I:553-566.

32. Dunne JB, Alexander B, Williams R, Tredger JM, Hoffman JL: Evidence that $\mathrm{S}$-adenosyl-L-methionine diastereoisomers may reduce ischaemia-reperfusion injury by interacting with purinoceptors in isolated rat liver Chromatographic analysis of the chiral and covalent instability of S-adenosyl-L-methionine. Br J Pharmacol 1998, I 25:225-233.
33. Hoffman JL: Chromatographic analysis of the chiral and covalent instability of S-adenosyl-L-methionine. Biochemistry 1986, 25:4444-4449.

34. Lee S, Floss HG, Gilbert BA, Rando RR: Steric Course of the Methyl Transfer from AdoMet to S-Farnesyl-3-thiopropionate by G-Protein Methyltransferase. J Org Chem 1998, 63:898-899.

35. Kealey JT, Lee S, Floss HG, Santi DV: Stereochemistry of methyl transfer catalyzed by tRNA (m5U54)-methyltransferase-evidence for a single displacement mechanism. Nucleic Acids Res 1991, 1 9:6465-6468.

36. Woodard RW, Tsai MD, Floss HG, Crooks PA, Coward JK: Stereochemical course of the transmethylation catalyzed by catechol O-methyltransferase. J Biol Chem 1980, 255:9|24-9| 27.

37. Borchardt RT, Wu YS: Potential inhibitors of S-adenosylmethionine-dependent methyltransferases. 5. Role of the asymmetric sulfonium pole in the enzymatic binding of $\mathbf{S}$ adenosyl-L-methionine. I Med Chem 1976, 19:1099-I I03.

38. Tredger JM: S-ADENOSYLMETHIONINE IN ISCHEMIAREPERFUSION INJURY: EXPERIMENTAL BASIS AND CLINICAL FINDINGS. 4th Reunion of Metabolism of Methionine Volume 15. Sierra Nevada, Granda, Spain; 1998.

\section{Pre-publication history}

The pre-publication history for this paper can be accessed here:

http://www.biomedcentral.com/1471-2474/5/6/prepub
Publish with Bio Med Central and every scientist can read your work free of charge

"BioMed Central will be the most significant development for disseminating the results of biomedical research in our lifetime. "

Sir Paul Nurse, Cancer Research UK

Your research papers will be:

- available free of charge to the entire biomedical community

- peer reviewed and published immediately upon acceptance

- cited in PubMed and archived on PubMed Central

- yours - you keep the copyright
BioMedcentral 